\title{
Cardiac Arrest with Extreme Hyperkalemia
}

\author{
Yasuharu Tokuda \\ Muribushi Okinawa Project for Teaching Hospitals, Okinawa, Japan
}

Cite this article as: Tokuda Y. Cardiac Arrest with Extreme Hyperkalemia. Eurasian J Emerg Med 2017; 16: $173-4$.

\section{Case Description}

A 34-year-old man with type 1 diabetes, which is being treated with a subcutaneous insulin self-injection, presented to the emergency department in a state of coma. The patient had skipped taking the insulin injection for four days and then complained of fatigue and epigastralgia. On the day of hospital arrival, his mother found him unconscious in his room at home and an ambulance brought him to our hospital. At the time of arriving at the emergency department, he had cardiac arrest and his electrocardiogram (Figure 1a) revealed pulseless electrical activity. Cardiopulmonary resuscitation was immediately performed. His serum glucose concentration was 1.178 $\mathrm{mg} / \mathrm{dL}$, arterial blood $\mathrm{pH}$ was 6.69 , and serum potassium concentration was $10.6 \mathrm{mEq} / \mathrm{L}$.

A diagnosis of diabetic ketoacidosis with severe hyperkalemia was made $(1,2)$. As his electrocardiogram (Figure $1 \mathrm{~b}$ ) revealed ventricular fibrillation during resuscitation, electrical defibrillation was conducted, but his electrocardiogram showed pulseless electrical activity. Calcium gluconate, normal saline, insulin, and bicarbonate were administered. After approximately $1 \mathrm{~h}$ of chest compression and artificial ventilation, his potassium concentration reduced to 5.4 $\mathrm{mEq} / \mathrm{L}$ and his electrocardiogram revealed sinus rhythm restoration (Figure 2) with palpable peripheral pulses. He was discharged home uneventfully after receiving education about the importance of receiving a regular insulin injection during 14 days of admission.

\section{Discussion}

Patients with diabetic ketoacidosis have a risk of cardiac arrest because of electrolyte imbalance, particularly potassium imbalance. The rapid evaluation of electrocardiograms is key to the immediate diagnosis of life-threatening potassium disorders. As in this case, bradycardia along with an absent $\mathrm{P}$ wave, wide QRS complex, and peaked (tentorial) $T$ wave characterize hyperkalemia on the electrocardiogram. Our present case proved that cardiac arrest with extreme hyperkalemia (more than $10 \mathrm{mmol} / \mathrm{L}$ ) can be reversible by correcting the serum potassium concentration while continuing efforts, such as by providing cardiopulmonary resuscitation. Effective medications include calcium gluconate, insulin, bicarbonate, and saline infusions. Cardiopulmonary resuscitation should be continued in patients with hyperkalemia until serum potassium concentrations are corrected. 

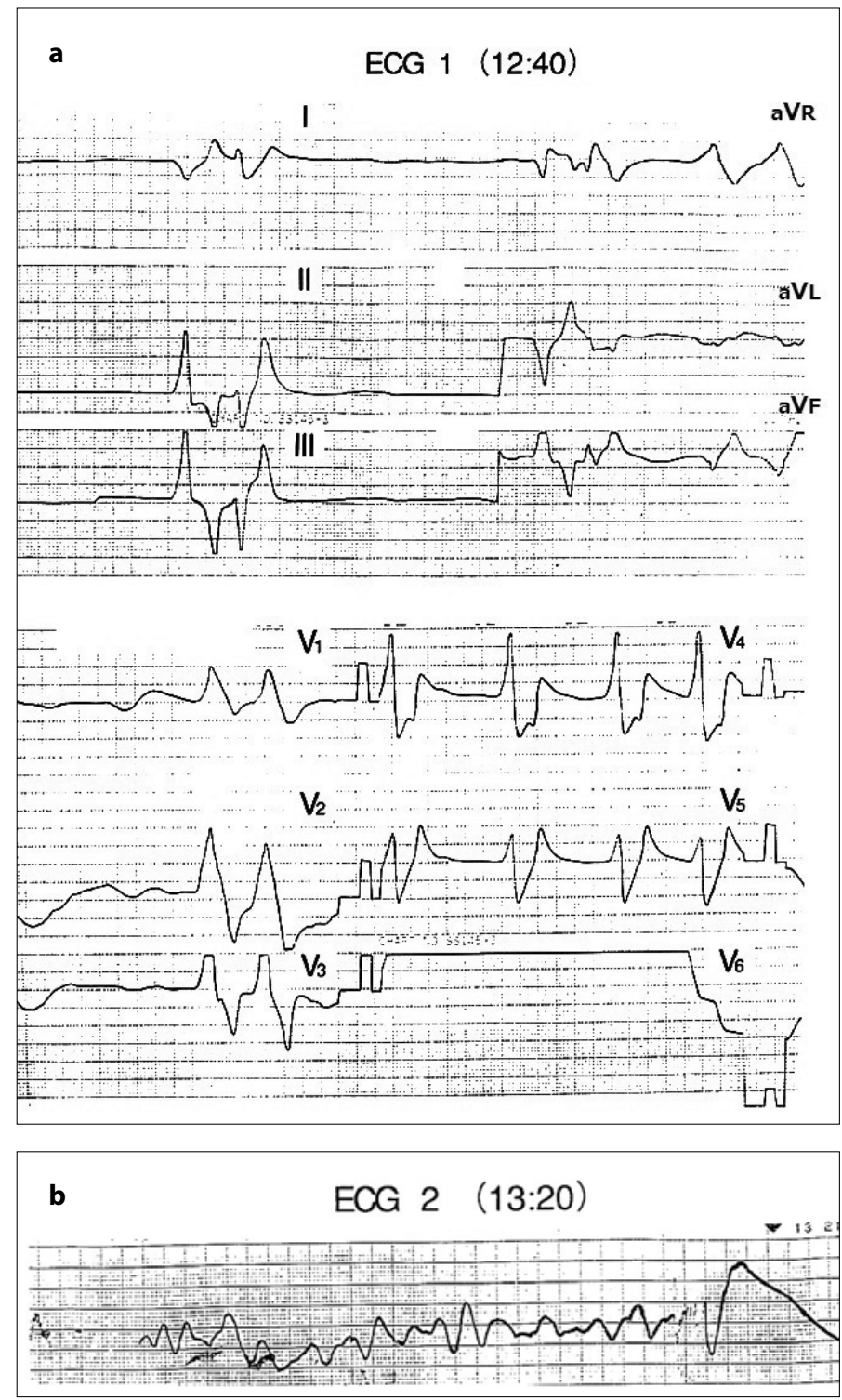

Figure 1. a, b. Initial electrocardiogram showing wave forms of pulseless electrical activity (lead V6 is not placed). (a) Monitored trace indicating ventricular fibrillation (b)

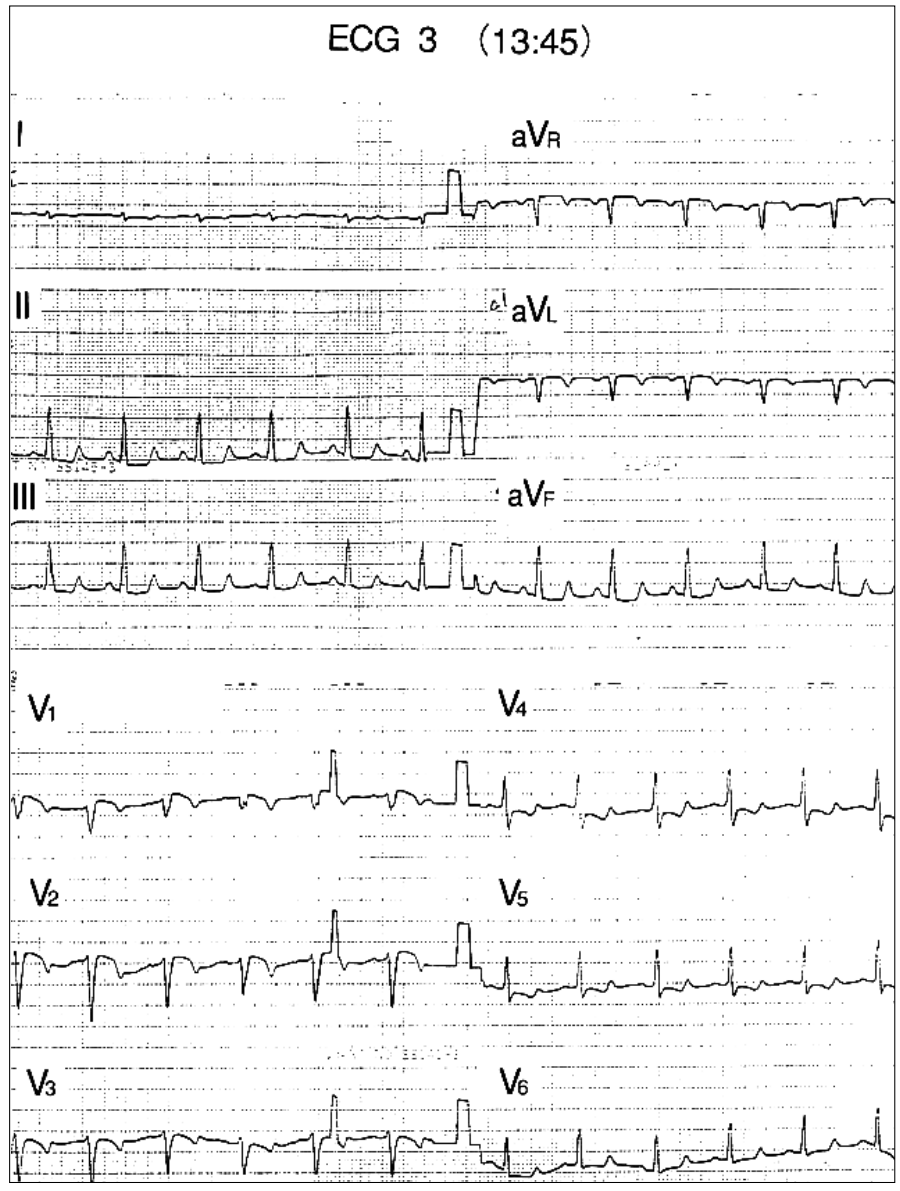

Figure 2. Follow-up electrocardiogram showing sinus rhythm with palpable peripheral pulses

\section{References}

1. Torrecilla C, de la Serna JL. Hyperkalaemic cardiac arrest, prolonged heart massage and simultaneous hemodialysis. Intensive Care Med 1989; 15: 325-6. [CrossRef]

2. Tran HA. Extreme Hyperkalemia. South Med J 2005; 98: 729-32. [CrossRef] 